\title{
Monitoring electrocardiograms of service users on high-dose methadone substitution therapy: an audit
}

\author{
Soumya Ghosh, ${ }^{1}$ Anil Kodagalli, ${ }^{1}$ Faiz Bhatti, ${ }^{1}$ Timothy Bradbeer ${ }^{1}$
}

The Psychiatrist (2010), 34, 489-491, doi: 10.1192/pb.bp.109.027813

'St. James' Hospital, Portsmouth Correspondence to Soumya Ghosh (drsoumya@indiatimes.com)

\begin{abstract}
Aims and method To explore the extent to which 2007 Department of Health guidelines on monitoring of high-dose ( $\geqslant 100 \mathrm{mg}$ ) methadone were followed, the reasons for non-adherence to these and the prevalence of QTc prolongation. We developed a simple tool for collecting data from case notes.

Results Out of 25 service users, 11 had had an electrocardiogram (ECG) and 7 had evidence of requests sent to general practitioners. After implementation of our recommendations, ten more service users had ECGs within 1 month. All but one ECG was normal. Methadone prescribing in favour of lower doses has been observed. QTc interval prolongation was not common.
\end{abstract}

Clinical implications Effective communication between primary and secondary care services is important in identifying cases and arranging ECGs.

Declaration of interest None.
Methadone was initially developed for pain relief but since 1960 it has been used as an opioid-substitution therapy to relieve narcotic craving, suppress abstinence symptoms and block the euphoric effects of heroin. To our knowledge, there was no systematic review available until $2003^{1}$ reporting the effective dose of methadone for substitution therapy. The organisation and regulation of methadone substitution therapy varied widely, which possibly left many service users on a wide range of doses of methadone.

QTc interval prolongation in electrocardiogram (ECG, above $500 \mathrm{~ms}$ ) can increase the risk of torsade de pointes, otherwise known as polymorphic ventricular tachycardia, with often fatal outcomes. ${ }^{2}$ There have been a number of reports published ${ }^{3-11}$ after the first report ${ }^{12}$ associating QTc interval prolongation and torsade de pointes with methadone use. Some of these reports showed that increase in QTc interval is dose related ${ }^{11,13}$ and high-dose methadone and concomitant use of agents that increase serum methadone levels are the most prevalent risk factors for torsade de pointes. ${ }^{14}$ Medicines and Healthcare Products Regulatory Agency guidelines ${ }^{15}$ have, therefore, recommended increased cardiac monitoring of service users who are on high-dose methadone $(\geqslant 100 \mathrm{mg} /$ day $)$ and with other risk factors, i.e. heart or liver disease, electrolyte abnormalities, and concomitant treatment with CYP 3A4 inhibitors. ${ }^{1,16}$ Although the Department of Health guidelines ${ }^{17}$ do not recommend blanket use of ECG for every service user, it advises that ECGs should be considered in users with the above risk factors. The aims of this audit were to explore the extent to which three substance misuse services adhered to the Department of Health guidelines for arranging ECGs for service users receiving high-dose methadone prescriptions. We also explored the reasons for non-adherence to guidelines (if any) and the prevalence of QTc interval prolongation.

\section{Method}

All service users being prescribed high-dose ( $\geqslant 100 \mathrm{mg} /$ day $)$ methadone by Portsmouth and South East Hampshire substance misuse services were included in this audit. These teams cover a city and its suburbs and are responsible for prescribing methadone to 580 service users. Names of service users were collected from the computerised database of the prescribing team. Two of the authors (S.G. and A.K.) collected the data (between June and July 2008) from service users' clinical notes using a simple datacollection tool. Data from September 2007 onwards was collected as Department of Health guidelines were updated at that time. The data collected included: demographic details of service users, present dose of methadone, duration of present dose prescribed, pre-existing cardiac conditions (if any), evidence of cardiac monitoring, date of last monitoring, findings of monitoring, professional/s involved in monitoring, how the results were communicated between services, the reasons for any communication failure and the measures taken to address it. At the beginning of the audit we decided to implement recommendations immediately following the presentation of the audit results. This allowed us to prospectively explore our hypothesised reasons for non-adherence to the guidelines.

\section{Results}

We identified 25 (4\%) service users (19 male, 6 female) on a wide range of high doses of methadone (100-180 mg daily) 
for a varying period of time ( 2 months to 30 years). Overall, $13(52 \%)$ service users were on $100 \mathrm{mg}$ methadone, 8 (32\%) on $120 \mathrm{mg}$ and 1 (4\%) each on 130, 140, 160 and $180 \mathrm{mg}$ daily (Table 1).

A total of 11 (44\%) service users were found to have had ECGs. Except for one ECG where the QTc interval was slightly increased $(444 \mathrm{~ms})$, all other ECGs were normal. However, this service user continued to receive a prescription for methadone. One service user had a history of myocardial infarction. Of the remaining 14 service users who had no record of having an ECG, 7 (50\%) had evidence of clinic letters sent to general practitioners (GPs) requesting them to arrange ECGs. For the other 7, no evidence of any communication from secondary care services was found. However, of these, two had been advised to contact their GPs to have an ECG.

\section{Adherence to recommendations}

This audit identified three factors leading to non-adherence to guidelines. First, for half the service users no referral was made by secondary care services to primary care services for an ECG. Second, for the other half, a referral was made by secondary care services to primary care services for an ECG but there was no record available in the individuals' notes showing that this had been carried out. Third, lack of motivation among service users may be a reason for ECGs not being carried out. Our recommendations were that clinicians and key workers should establish effective communication with primary care services, spread awareness about the guidelines and be proactive to increase the motivation of service users.

Audit findings with recommendations were presented at a joint meeting of all three services. In order to establish effective communication, key workers and clinicians were encouraged to liaise more closely with primary care services for arranging ECGs. This was achieved by ensuring that where indicated a referral for ECG was always made, and by making telephone calls to the GP surgery to follow up any referrals that had been made to ensure that the ECG is carried out. In addition to this, clinicians were asked to spread awareness of the guidelines among GPs where required and to inform them about their role in helping secondary care services to follow the guidelines. Key workers were asked to take a proactive role in motivating service users by helping them to understand the importance of attending their ECG appointment and in the case of more reluctant service users, to accompany them to surgeries. We received an extremely positive response from primary care services. Ten more service users had ECGs within 1 month; all 10 ECGs were normal.

\section{Discussion}

The audit showed that only a small proportion of service users (4\% of total prescription) were being prescribed highdose methadone; only 4 of whom were prescribed in excess of $120 \mathrm{mg}$ per day. The rest of the service users were on a methadone substitution therapy with a dose of methadone below $100 \mathrm{mg}$ per day.
Table 1 Basic demographics and outcomes

\begin{tabular}{lc} 
Characteristics & $n(\%)$ \\
\hline Service users, $n$ & $25(100)$ \\
\hline Male & $19(73)$ \\
\hline Dose of methadone, mg/day & \\
100 & $13(52)$ \\
120 & $8(32)$ \\
130 & $1(4)$ \\
140 & $1(4)$ \\
160 & $1(4)$ \\
180 & $1(4)$ \\
\hline Duration of present dose, years & \\
$0-1$ & $7(28)$ \\
$1-2$ & $10(40)$ \\
$2-5$ & $4(16)$ \\
$>5$ & $3(12)$ \\
Not found & $1(4)$ \\
\hline ECGs, total & $21(84)$ \\
Before implementation of recommendations, $n$ & $11(52)$ \\
After implementation of recommendations, $n$ & $10(48)$ \\
\hline ECG results & \\
Normal & $20(95)$ \\
QTc prolongation (444 ms) & $1(5)$ \\
\hline Existing cardiac problems & $1(4)$ \\
History of myocardial infarction & $7(50)$ \\
\hline Communication with GPs requesting ECG & \\
(before implementation of audit recommendations) & \\
Clinical letters sent to GPs & \\
No record of communication & \\
\hline
\end{tabular}

ECG, electrocardiogram; GP, general practitioner.

Audit findings revealed that non-adherence to guidelines was often as a result of a lack of effective communication between primary and secondary care services in the catchment area of these three services. Substantial improvement in adherence to guidelines following the implementation of our recommendations confirmed that the factors identified in the audit were the reasons for non-adherence.

The three substance misuse services in this audit are dependent on primary care services for the arrangement and interpretation of ECGs. This is because they are neither equipped to carry out ECGs nor do they have adequate expertise to interpret them. Substance misuse teams are usually led by a consultant psychiatrist with very little or no direct input from other specialists. A recent survey showed that only 5\% of trainees and consultant psychiatrists could correctly indicate QTc intervals. ${ }^{18}$ Computer-assisted ECG interpretation is not completely reliable ${ }^{18}$ and errors are common, ${ }^{19}$ so interpretation should not be accepted without a visual inspection of the ECG, preferably by an expert. $^{19}$ Therefore, in order to adhere to guidelines, psychiatrists in substance misuse teams would need to have expertise in ECG interpretation. This would require making basic training of reading ECG tracing mandatory for psychiatrists at all levels; particularly for psychiatric trainees. ${ }^{18,20}$ The other option would be to provide easy access to cardiologists ${ }^{20}$ or GPs with special interest in cardiology. As indicated by the findings, the other possible solution is to increase collective commitment and effective 
communication between primary and secondary care services. The number of primary care services prescribing substitution medication is growing. ${ }^{21,22}$ This, we hope will increase contact between service users and their GPs. It could possibly ensure that most of the issues related to drug use, including cardiac monitoring as per the guidelines, will be successfully managed in general practice. The difficulty of non-adherence to the guidelines may become less of an issue in the future.

The ECG results in our audit showed that the prevalence of QTc interval prolongation was very low among the service users. However, the result does not deflect from the importance of taking a cautious approach before prescribing high-dose methadone. We find the recently published cardiac safety recommendations ${ }^{14}$ to be highly appropriate. These are: informing service users about the risk of arrhythmia; taking a clinical history of structural heart problems, arrhythmia and syncope; obtaining ECGs for all service users before prescribing methadone; considering discontinuation and reduction of dose in individuals with QTc prolongation $(>500 \mathrm{~ms})$; elimination of risk factors; and being aware of drugs that interact with methadone.

\section{Limitations}

Other risk factors contributing to QTc interval prolongation were not recorded in this audit. However, normal ECG findings indirectly ruled out the contribution of these factors in prolonging QTc interval. The number of service users included in the audit is small and there was a relatively small number of service users on a very high dose of methadone. Nevertheless, we were able to identify areas requiring improvement in order to provide the standard of care compliant with the published standards.

\section{About the authors}

Soumya Ghosh and Anil Kodagalli are specialty trainees in the Substance Misuse Service at St James' Hospital, Portsmouth. Faiz Bhatti and Timothy Bradbeer are consultant psychiatrists in the Substance Misuse Service at St James' Hospital Portsmouth.

\section{References}

1 Faggiano F, Vigna-Taglianti F, Versino E, Lemma P. Methadone maintenance at different dosages for opioid dependence. Cochrane Database Syst Rev 2003; 3: CD002208.

2 Botstein P. Is QT interval prolongation harmful? A regulatory perspective. Am J Cardiol 1993; 72: 50B-52B.

3 De Bels D, Staroukine $M$, Devriendt J. Torsades de pointes due to methadone (Letter). Ann Intern Med 2003; 139: E156.
4 Sala M, Anguera I, Cervantes M. Torsade de points due to methadone (Letter). Ann Intern Med 2003; 139: W64.

5 Gil M, Sala M, Anguera I, Chapinal O, Cervantes M, Guma JR, et al. QT prolongation and torsades de pointes in patients infected with human immunodeficiency virus and treated with methadone. Am J Cardiol 2003; 92: 995-7.

6 Decerf JA, Gressens B, Brohet C, Liolios P. Can methadone prolong QT interval? (Letter). Intensive Care Med 2004; 30: 1690-1.

7 Almehmi A, Malas AM, Yousufuddin M, Rosencrance JG. Methadoneinduced torsade de pointes in a patient with baseline QT interval. $W \mathrm{~V}$ Med J 2004; 100: 147-8.

8 Sticherling C, Schaer BA, Ammann P, Maeder M, Osswald S. Methadone-induced torsade de pointes tachycardias. Swiss Med Wkly 2005; 135: 282-5

9 Peles E, Bodner G, Kreek MJ, Rados V, Adelson M. Corrected-QT intervals as related to methadone dose and serum level in methadone maintenance treatment (MMT) patients - a cross-sectional study. Addiction 2007; 102: 289-300

10 Routhier DD, Katz KD, Brooks DE. QTc prolongation and torsades de pointes associated with methadone therapy. J Emerg Med 2007; 32 275-8.

11 Krantz MJ, Kutinsky IB, Robertson AD, Mehler PS. Dose-related effects of methadone on qt prolongation in a series of patients with torsade de pointes. Pharmacaother 2003; 23: 802-5.

12 Krantz MJ, Lewkowiez L, Hays H, Woodroffe MA, Robertson AD, Mehler PS. Torsade de Pointes associated with very-high-dose methadone. Ann Intern Med 2002 : 137: 501-4.

13 Krantz MJ, Martin J, Stimmel B, Mehta B, Haigney MCP. QTc Interval screening in methadone treatment. Ann Intern Med 2009; 150: 387-95.

14 Justo D, Gal-Oz A, Paren Y, Goldin Y, Zeltser D. Methadone-associated Torsades de Pointes (polymorphic ventricular tachycardia) in opioiddependent patients. Addiction 2006; 101: 1333-8.

15 Medicine and Healthcare Products Regulatory Agency. Current Problems in Pharmacovigilance (Vol 31). MHRA, 2006

16 Ehret GB, Voide C, Gex-Fabry M, Chabert J, Shah D, Broers B, et al Drug-induced long QT syndrome in injection drug users receiving methadone. Arch Intern Med 2006; 166: 1280-7.

17 Department of Health and the devolved administrations. Drug Misuse and Dependence: UK Guidelines on Clinical Management. Department of Health (England), the Scottish Government, Welsh Assembly Government and Northern Ireland Executive, 2007.

18 Solomons L, Treloar A, Noronha R. Competence of psychiatric clinicians in interpreting electrocardiograms and QT intervals: can they do this? Does it matter? Psychiatr Bull 2008; 32: 291-4.

19 British Heart Foundation. Factfile 12 Computer-Assisted ECG Interpretation. British Heart Foundation, 2005 (http://www.bhf.org.uk/ research_health_professionals/health_professionals/factfiles.aspx).

20 Royal College of Psychiatrists. Consensus Statement on High-Dose Antipsychotic Medication. Council Report (CR138). Royal College of Psychiatrists, 2006 (http://www.rcpsych.ac.uk/files/pdfversion/ CR138.pdf).

21 National Treatment Agency for Substance Misuse. Effective Treatment Changing Lives. Regional Teams: South East. NTA, 2009 (http:// www.nta.nhs.uk/regional.aspx).

22 Ford C. Supporting GP's in improving substitute prescribing for opiate users in UK General Practice. Heroin Addiction Relat Clin Probl 2007; 9: 31-40. 